\title{
Mass Screening of Vision in the Cinemas and a Maculascope for the Masses: Revival of Haidinger's Brush
}

\author{
Kodikullam V. Avudainayagam and Chitralekha S. Avudainayagam \\ School of Optometry and Vision Science, UNSW Australia, Sydney, NSW 2052, Australia \\ Correspondence should be addressed to Kodikullam V. Avudainayagam; avudaikv@gmail.com
}

Received 19 October 2014; Revised 16 December 2014; Accepted 16 December 2014; Published 31 December 2014

Academic Editor: Joseph Rosen

Copyright ( $) 2014$ K. V. Avudainayagam and C. S. Avudainayagam. This is an open access article distributed under the Creative Commons Attribution License, which permits unrestricted use, distribution, and reproduction in any medium, provided the original work is properly cited.

\begin{abstract}
Haidinger's brush, an entoptic phenomenon, is very useful in checking central vision loss and eccentric fixation. Haidinger's brush is also used to provide vision training for subjects affected by eccentric fixation/lazy eye. We propose the use of Haidinger's brush for mass self-screening of vision in cinema theatres in one go and at no cost to the subjects. We also suggest a simple projection technology for mass vision screening of subjects in waiting rooms or at shop windows of eye-care practitioners. Finally, we propose a new affordable handheld device for vision training. The test and the devices that we propose would increase public awareness of eye health, improve vision in children, and help prevent blindness in the elderly.
\end{abstract}

\section{Introduction}

Haidinger's brush is an entoptic phenomenon which was first observed and reported by von Haidinger in 1844 [1]. It is based on our sensitivity to polarised light. When we look at the blue sky suddenly, we perceive a yellow brush like pattern in the shape of a bow tie which quickly fades away due to adaptation. If we look at a white background through a slowly rotating polariser we would see rotating yellow and blue brush-like patterns that remain perpendicular to each other. The yellow brushes when viewed through a blue filter appear as dark brushes in a blue background and are more easily perceived. The appearance of the dark brushes as seen by a subject is shown in Figure 1. The theory behind the formation of these fringes is still a topic of research [2]. Haidinger's brush is formed only on the macula and centred on the fovea. Hence, for subjects having normal vision it appears centred on the object of fixation. In eccentric fixation (EF) used by subjects who have some problem with their central vision, Haidinger's brush appears away from the object of fixation. Thus Haidinger's brush is very useful to test for EF in growing children when their vision is developing $[3,4]$. In macular degeneration (MD) Haidinger's brush pattern would appear disrupted. Hence Haidinger's brush is very useful in the diagnosis of MD for the elderly population $[3,4]$.
Its clinical use for testing macular function was recognised in 1950 when Goldscmidt developed a "Macular Deficiency Tester" in which he made use of a rotating polariser that enabled prolonged viewing of Haidinger's brushes [5]. An interesting feature of these brushes is that it is possible for cataractous patients to see it which is of great value to confirm macular function preoperatively [6]. Its clinical use for eccentric fixation was promoted by Dr. Curt Cüppers of Geissen who designed a coordinator for the diagnosis and treatment of eccentric fixation $[6,7]$.

In recent times, scanning laser polarimetry is used to study the integrity of the macula through objective observation of the bow-tie pattern [8]. While disruption of the bow-tie pattern in the scanning laser polarimetry images of the fovea indicates macular degeneration, regular bowtie patterns may be obtained even though there may be some underlying pathology [9]. Subjective observation of Haidinger's brushes on the other hand would have the potential to reveal some underlying pathologies early.

Currently, in the clinic, a bench-top device, the Macula Integrity Tester (MIT) is used to help subjects see Haidinger's brush [10]. The test is carried out on an individual by the practitioner. Only some practitioners have the provision to carry out this test. Further, for vision training, the subjects visit the practitioner. 


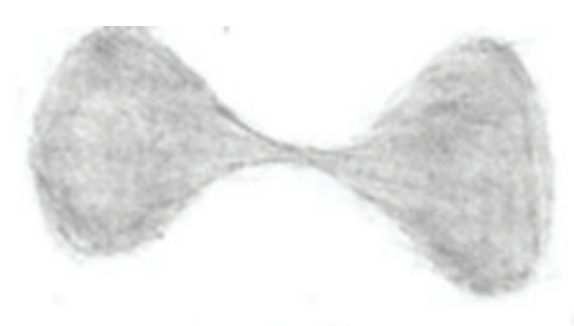

FIGURE 1: Sketch of Haidinger's brush as seen by a subject.

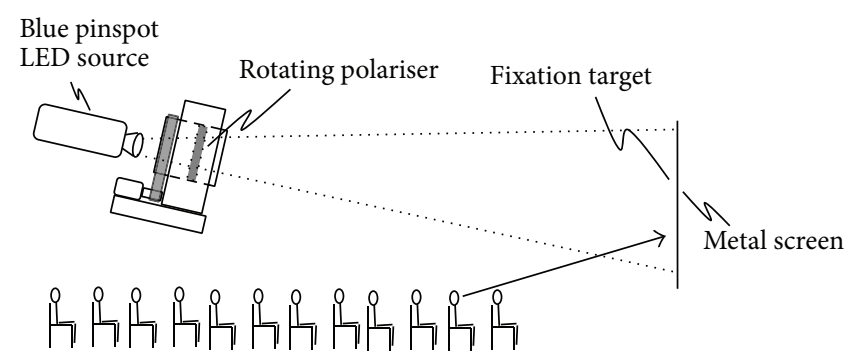

FIGURE 2: Projection of Haidinger's brush for mass screening in a theatre.

In this paper, we propose projection of Haidinger's brush in cinema theatres for screening a large number of subjects in one go and at no cost to them. We also propose a simple technology for projecting Haidinger's brush in waiting rooms/shop windows of eye-care practitioners for self-test of individuals visiting the clinic. We also propose a technology that can provide a simple low cost hand held device for the use of practitioners on subjects as well as for the use of individuals for vision training.

\section{Mass Self-Screening of Vision}

2.1. Mass Self-Screening of Vision in the Cinemas. For the vision screening test in the cinemas we propose an LED based projection unit to project Haidinger's brush on the metallic screen that is used for 3D movies (see Figure 2).

The projection unit consists of a rotating polariser placed in front of a blue pinspot LED source ( $3 \mathrm{~W}$ Blue LED Source-Mini Spotlight-SL-3471 (CREE LED) - 97(L) $\mathrm{mm} \times$ 56(Dia) $\mathrm{mm}$ ) as shown in Figure 3.

The light from the projection unit illuminates the polarisation maintaining metallic screen used in the cinemas. Along with the projection of light with rotating polarisation, a fixation target (picture of a dot) will be projected on the metallic screen using the normal cinema projector. The audience will be instructed through an announcement to fixate on the dot monocularly and note if a rotating sand clock/bowtie pattern (Haidinger's brush) is seen centred on the dot. Individuals with normal vision will see Haidinger's brush centred on the dot. Individuals with eccentric fixation will see Haidinger's brush off-centred from the dot. Haidinger's brush will appear ill-defined/disrupted for individuals with macular degeneration and they will have difficulty seeing it. Subjects, for whom the pattern is not seen well defined or is seen offcentred from the dot when looking at the dot, will be advised to consult an optometrist/GP/eye-care professional.
2.2. Simple Technology for the Projection of Haidinger's Brush in Waiting Rooms/Shop Windows of Eye-Care Practitioners. A sketch of Haidinger's brush projection unit for a self-test, for subjects visiting the eye-care clinic, is given in Figure 4.

Four high power, blue pinspot LEDs are used to illuminate the same area of the metal screen of the cinemas. Each LED is placed behind a linear polariser. The transmission axes of the four polarisers are made to be vertical, $45^{\circ}$, horizontal, and $135^{\circ}$, respectively. Using a switching circuit the LEDs are switched on in succession for $125 \mathrm{~ms}$ every half a second. This will simulate the effect of a rotating polariser in front of a single LED source. This unit would be easier to fabricate and maintain as it obviates the need to have a motor to rotate the polariser. To ensure that the light from all the LEDs overlaps perfectly on the screen the LEDs could illuminate a field stop that is projected onto the screen. This will alleviate the need to align the LEDs.

Additional advantages offered by this technology are that it is a simple low cost device that can be packaged into a display box unit with a back projection screen and used for vision screening of the public waiting at the practice of an optometrist/ophthalmologist/GP along with audio/text instructions. Such units can also be used, for example, at the optometrists' shop windows.

\section{The AC Maculascope}

The AC maculascope is a proposed new device to check for macular degeneration/eccentric fixation in subjects, using Haidinger's brush. AC here stands for Avudai-Chitra, the authors who are the inventors of this device. It is a simple, lightweight, inexpensive handheld device that can be used by eye-care professionals and by individuals for vision training if required. The device that is currently in use (MIT) makes use of a polariser, a motor to rotate the polarizer, a blue filter, and a $40 \mathrm{~W}$ bulb [10]. Another version of the MIT makes 


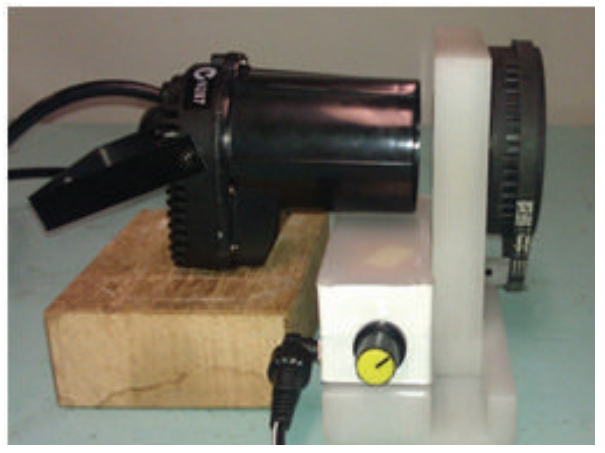

FIGURE 3: Arrangement to produce linearly polarized light with the direction of polarization rotated uniformly at $60 \mathrm{rev} / \mathrm{min}$.
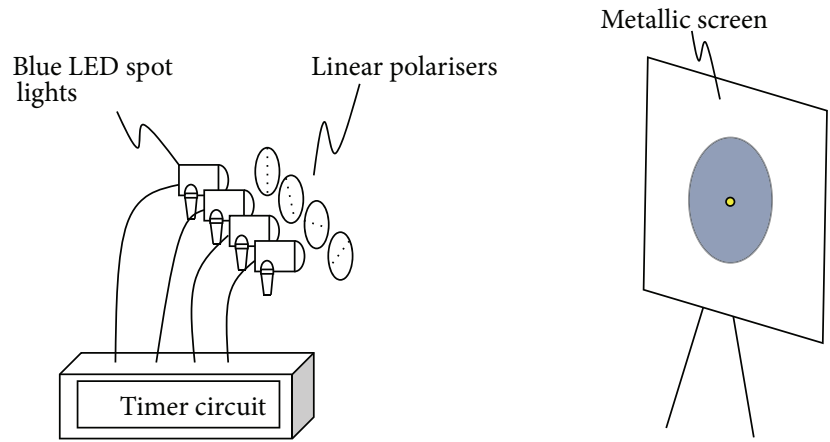

FIGURE 4: Alternate technology for projecting Haidinger's brush for several subjects. The individual fixating on the yellow dot will see a rotating Haidinger's brush as per the quality of his/her central vision.

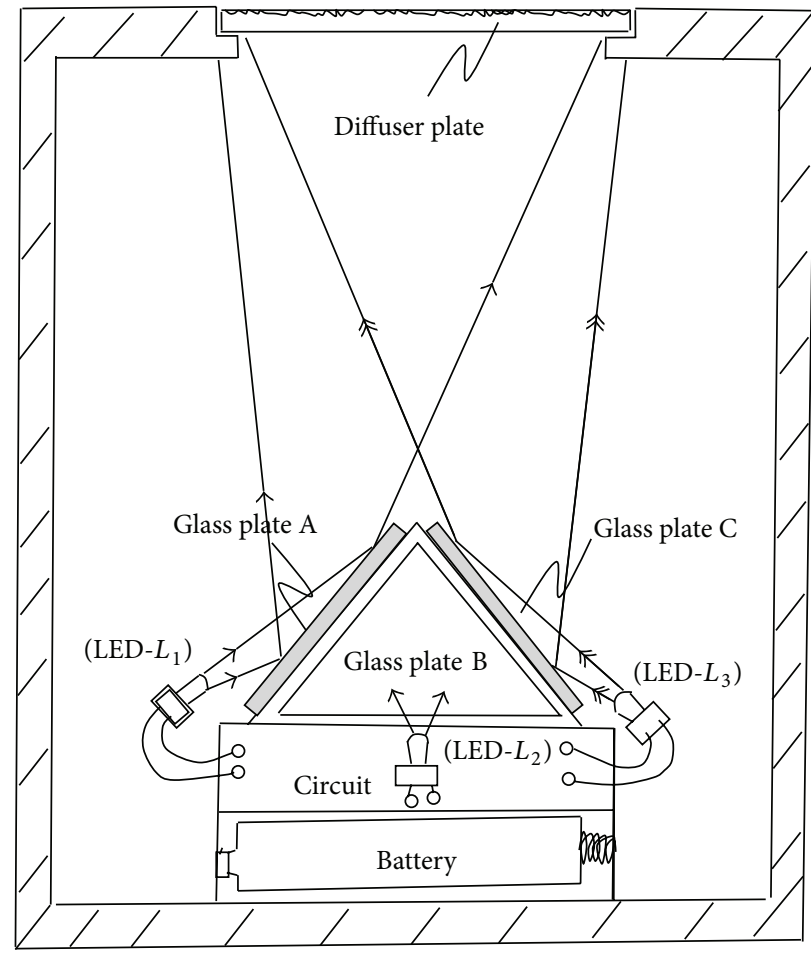

Figure 5: The AC maculascope. 


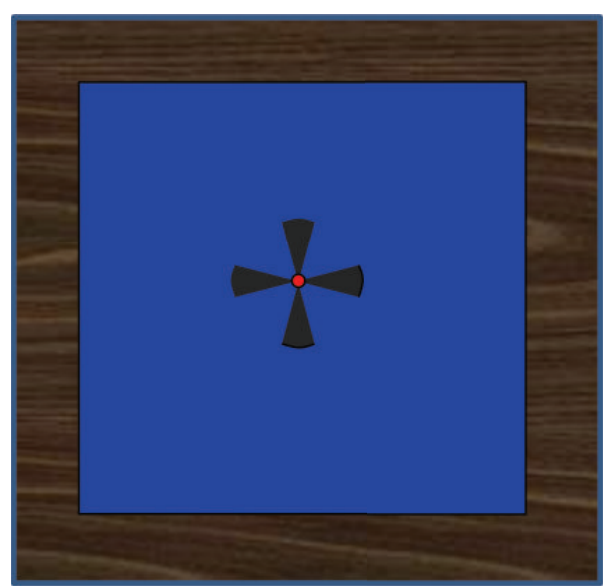

(a)

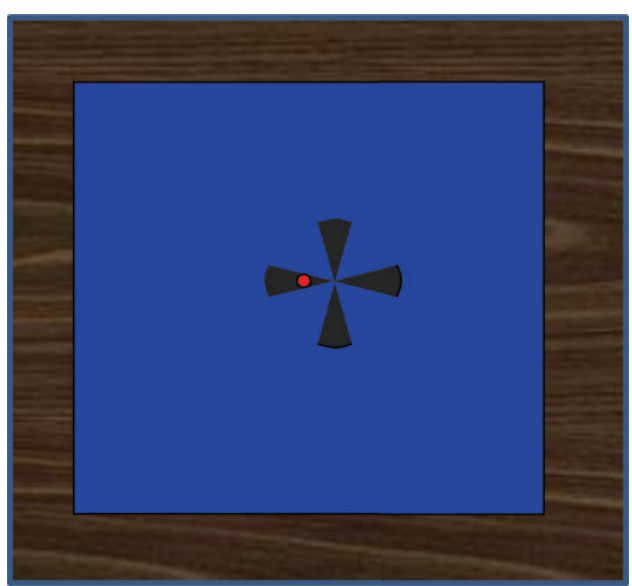

(b)

FIGURE 6: (a) View obtained by a normal subject. (b) View obtained by a subject having eccentric fixation.

use of a light bulb, a liquid crystal unit to rotate the plane of polarisation of the light, and a blue filter [10]. The proposed AC maculascope does not make use of a polariser, motor, liquid crystal, blue filter, or the light bulb. It operates on a very simple principle and makes use of LEDs, glass plates, a simple switching circuit, and a diffuser screen. Polarised light in our device is generated by reflection of light from the glass plates at the Brewster angle. Bright blue LED sources are used to illuminate the glass plates. This obviates the need for a blue filter.

The device is shown in Figure 5. A square pyramid serves as the mounting base for four glass plates. The LEDs used (Chrome Bezel with $5 \mathrm{~mm}$ Blue LED) to illuminate the glass plates that are opposite to each other are switched on simultaneously. LEDs 1 and 3 are switched on simultaneously to illuminate glass plates $\mathrm{A}$ and $\mathrm{C}$ and LEDs 2 and 4 are switched on simultaneously to illuminate glass plates B and D (LED 4 and glass plate D are not seen in Figure 5). The switching of LEDs 1 and 3 alternates and succeeds the switching of LEDs 2 and 4 . The switching speed is around 60 times a min. The glass plates are angled such that the angle of incidence of the LED light on the glass plate corresponds to the Brewster angle of incidence and the reflected light is then largely linearly polarised. The authors used flint glass of refractive index 1.6, for which the Brewster angle is $58^{\circ}$. The glass pyramid + LEDs are inside a light tight box that has a diffuser glass plate as the top surface. Linearly polarised light from plates A, B, C, and $D$ illuminates the diffuser screen face of the box. The state (orientation) of polarisation of the light from $\mathrm{A}$ and $\mathrm{C}$ will be along the length of the diffuser lid and the state of polarisation of the light from $\mathrm{B}$ and $\mathrm{D}$ will be along the width of the lid. Haidinger's brush pattern seen will therefore be in the form of a cross. In principle LEDs 1 and 2 would suffice. The extra pair is to generate a more uniform and brighter illumination of the diffuser plate. A fixation dot is provided at the centre of the diffuser screen to enable subjects to fixate. When the LEDs are switched on, the view obtained by a normal subject is shown in Figure 6(a). Figure 6(b) shows the appearance of the cross for a subject who has a fixation problem. The cross will appear shifted from the fixation dot if the subject has a fixation problem. The cross will appear disrupted for a subject who is suffering from macular degeneration. The LEDs can be battery operated making the device light weight and hand held.

This concept could be extended by using LEDs along greater number of meridians to generate a rotating Haidinger's brush.

\section{Conclusions}

We have come up with an innovation for mass self-screening of vision which will facilitate timely detection of macular degeneration and diagnosis of eccentric fixation for a large number of subjects in one go and at no cost to them. It will help in monitoring eye health in the elderly and vision development in children. Screening fixation problems in school children will promote full development of their vision. This innovation will increase public awareness of eye health and prevention of blindness. Additional spin-offs include community service and scope to attract advertisement revenue for governments.

The newly proposed handheld device would be a low cost testing device that would be easier for eye-care practitioners to acquire. Patients will also be able to readily afford one for vision training if required. The device is battery operated with very low power consumption. It would be handheld, lightweight, and compact making it convenient for use.

The devices proposed here could be used as valuable teaching aids in Schools of Optometry/Medicine.

The devices reported here have been fabricated and found to work well.

\section{Conflict of Interests}

The authors declare that there is no conflict of interests regarding the publication of this paper.

\section{Acknowledgments}

The authors would like to thank Professor Les Field (DVCResearch), UNSW Australia, for his encouragement and Mr. 
Dale Arnul of Wilson and Gilkes, Australia, for lending the metallic screen unconditionally for research.

\section{References}

[1] W. K. von Haidinger, "Ueber das direct Erkennen des polarisierten lichtes und der Lage der Polarisations Ebene," Annalen der Physik und Chemie, vol. 63, pp. 29-39, 1844.

[2] A. le Floch, G. Ropars, J. Enoch, and V. Lakshminarayanan, "The polarization sense in human vision," Vision Research, vol. 50, no. 20, pp. 2048-2054, 2010.

[3] A. H. Tunnacliffe, Introduction to Visual Optics, Association of British Dispensing Opticians, 4th edition, 2004.

[4] R. B. Rabbetts, Bennett and Rabbetts' Clinical Visual Optics, Butterworth Heinemann Elsvier, New York, NY, USA, 4th edition, 2007.

[5] M. Goldscmidt, "A new test for function of the macula lutea," Archives of ophthalmology, vol. 44, no. 1, pp. 129-135, 1950.

[6] M. E. Sherman and B. S. Priestley, "The Haidinger brush phenomenon. A new clinical use," American Journal of Ophthalmology, vol. 54, no. 5, pp. 807-812, 1962.

[7] B. E. Vodnoy, "Utilization of Haidinger brushes in orthoptics," American Journal of Optometry and Archives of American Academy of Optometry, vol. 39, pp. 543-547, 1962.

[8] A. Katsanos, P. Kóthy, A. Papp, and G. Holló, "Influence of subfoveal choroidal neovascularisation on macular imaging with scanning laser polarimetry of the retinal nerve fibre layer," Eye, vol. 19, no. 2, pp. 117-122, 2005.

[9] A. Weber, A. E. Elsner, M. Miura, S. Kompa, and M. C. Cheney, "Relationship between foveal birefringence and visual acuity in neovascular age-related macular degeneration," Eye, vol. 21, no. 3, pp. 353-361, 2007.

[10] Macula Integrity Tester (MIT), http://www.bernell.com/product/3271/184. 

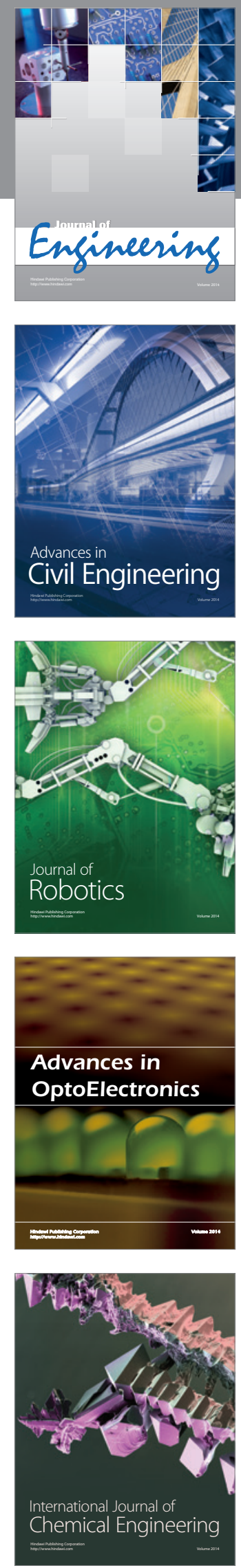

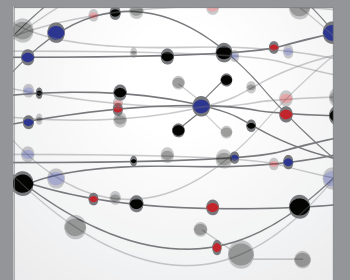

The Scientific World Journal
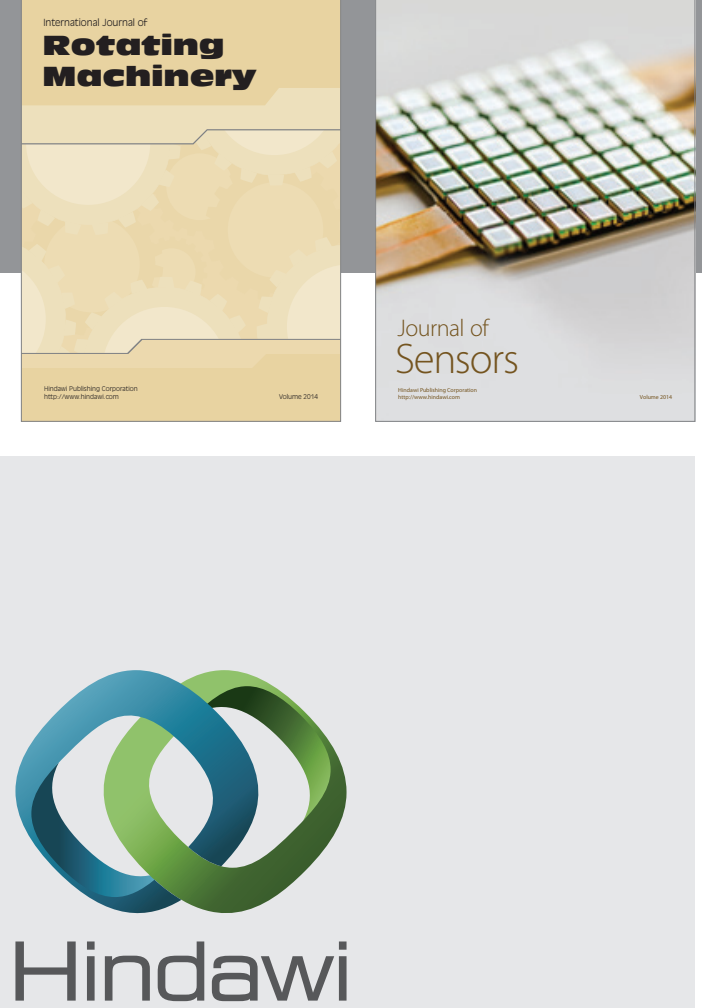

Submit your manuscripts at http://www.hindawi.com
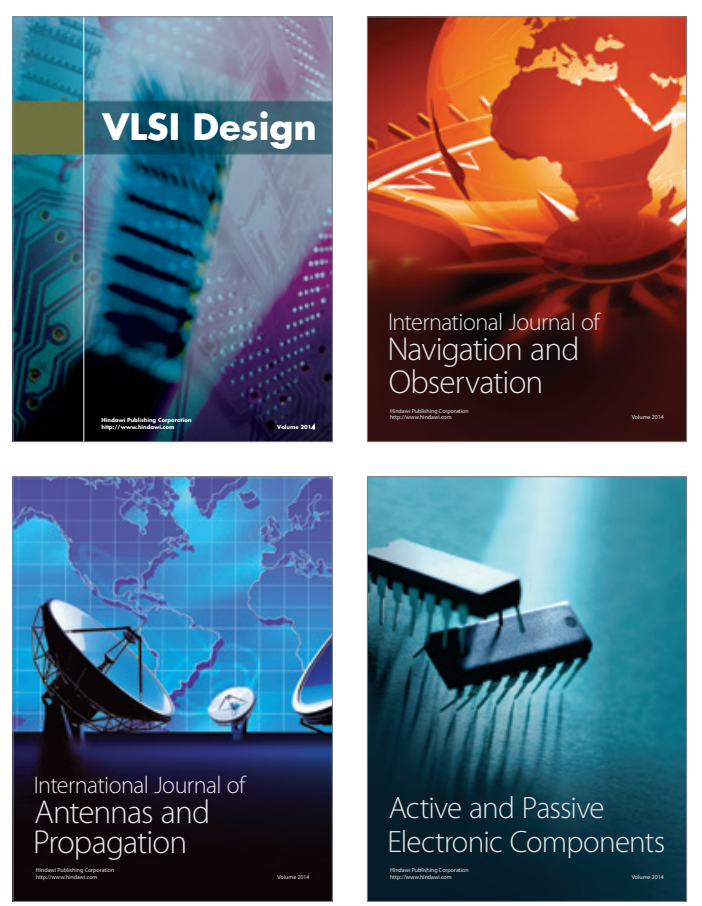
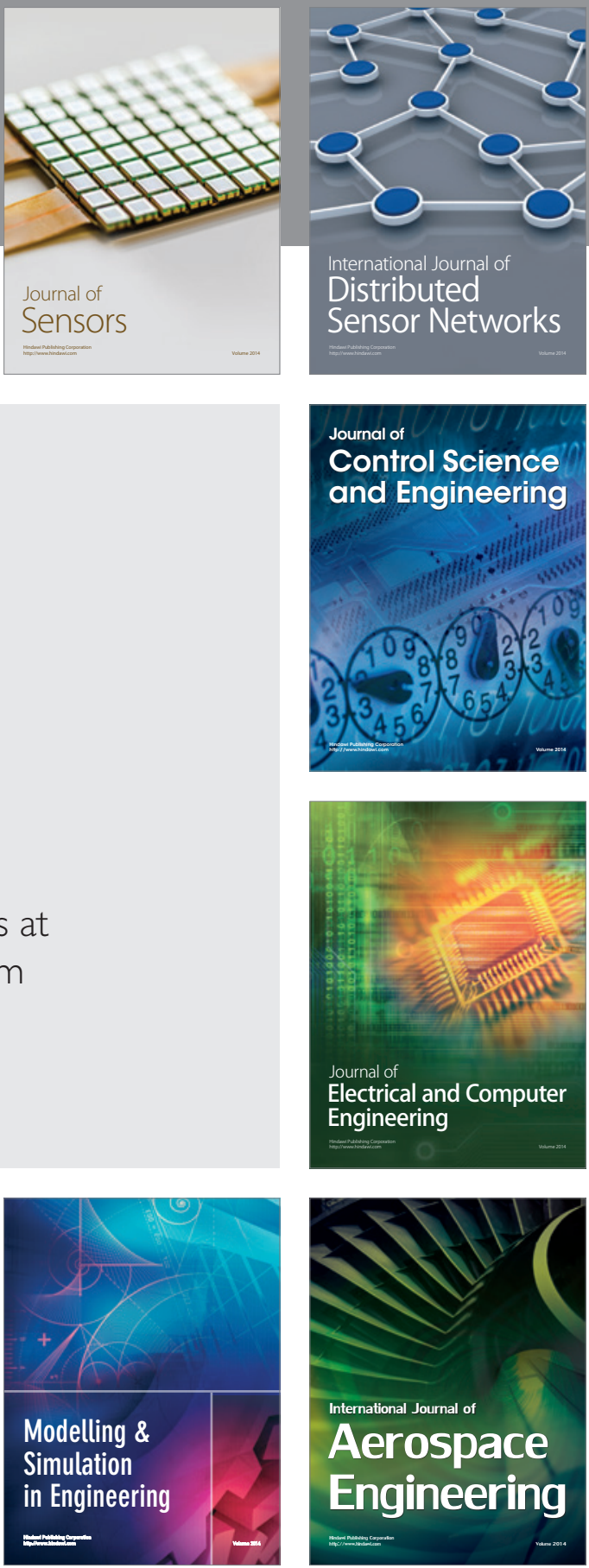

Journal of

Control Science

and Engineering
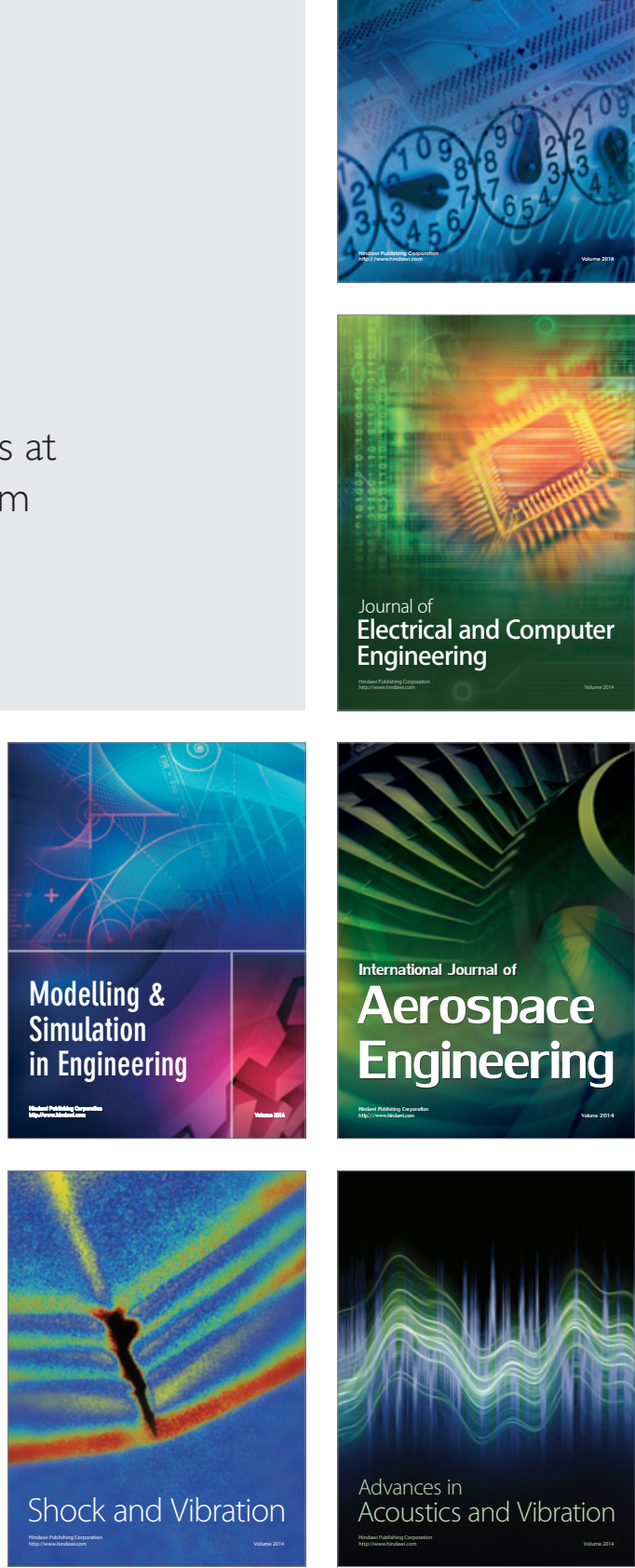